Herzschr Elektrophys 2016 $27: 171-185$

DOI 10.1007/s00399-016-0449-z

Received: 15 June 2015

Accepted: 2 August 2016

Published online: 30 August 2016

๑) Springer-Verlag Berlin Heidelberg 2016

CrossMark
Thomas Brüggemann' - Daniel Dahlke ${ }^{2}$ Amin Chebbo' • Ilka Neumann'

'Global Education and Training, Cardiac Rhythm Management, BIOTRONIK SE \& Co. KG, Berlin, Germany

${ }^{2}$ Technical Services, BIOTRONIK Inc., Lake Oswigo, USA

\section{Tachycardia detection in modern implantable cardioverter-defibrillators}

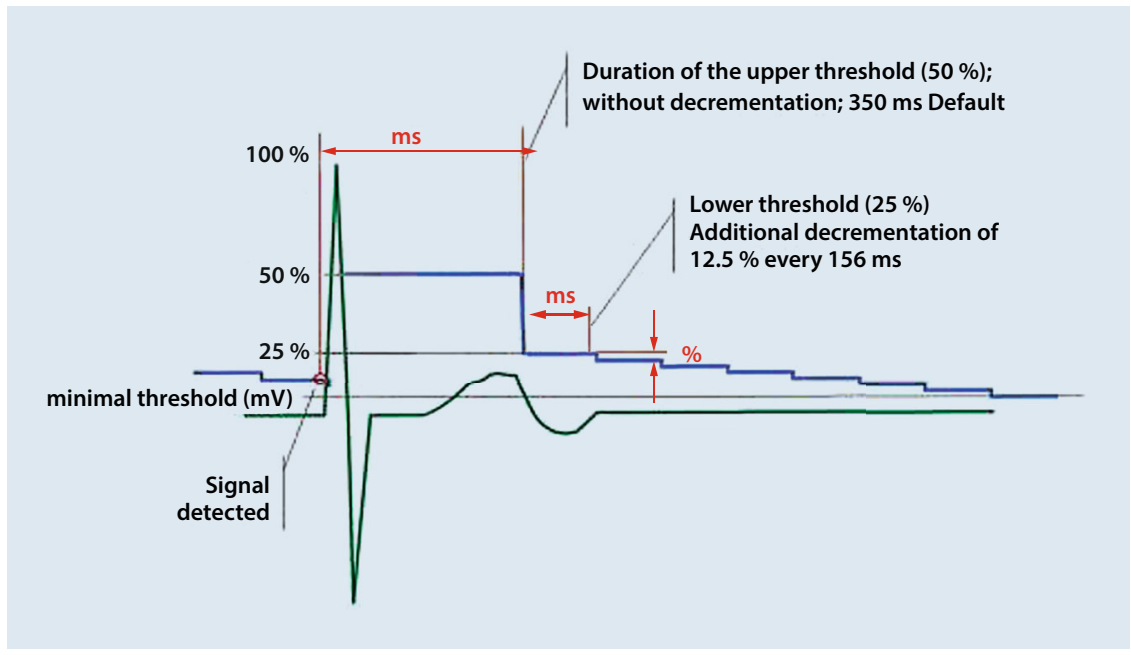

Fig. $1 \Delta$ Automatic sensitivity control with standard values for RV sensing: the R-wave is tracked and determined to be $16.0 \mathrm{mV}$ at its peak. The amplitude is measured in the first $110 \mathrm{~ms}$ of the complex. Following the sensed event the upper threshold is set to $8.0 \mathrm{mV}$ (i. e., $50 \%$ of the R-wave amplitude). At the end of the upper threshold duration of $350 \mathrm{~ms}$ the device decreases the sensing threshold to $4.0 \mathrm{mV}$ (i. e., $25 \%$ of the R-wave amplitude). It remains at the lower threshold for $156 \mathrm{~ms}$ and then decrements by $12.5 \%$ every 156 ms afterward until the minimum threshold is reached. The $12.5 \%$ decrement is measured from the previous setting, for example, $4.0 \times 87.5 \%=3.5 \mathrm{mV}, 3.5 \times 87.5 \%=3.1 \mathrm{mV}, 3.1 \times$ $87.5 \%=2.7 \mathrm{mV}$ and so on

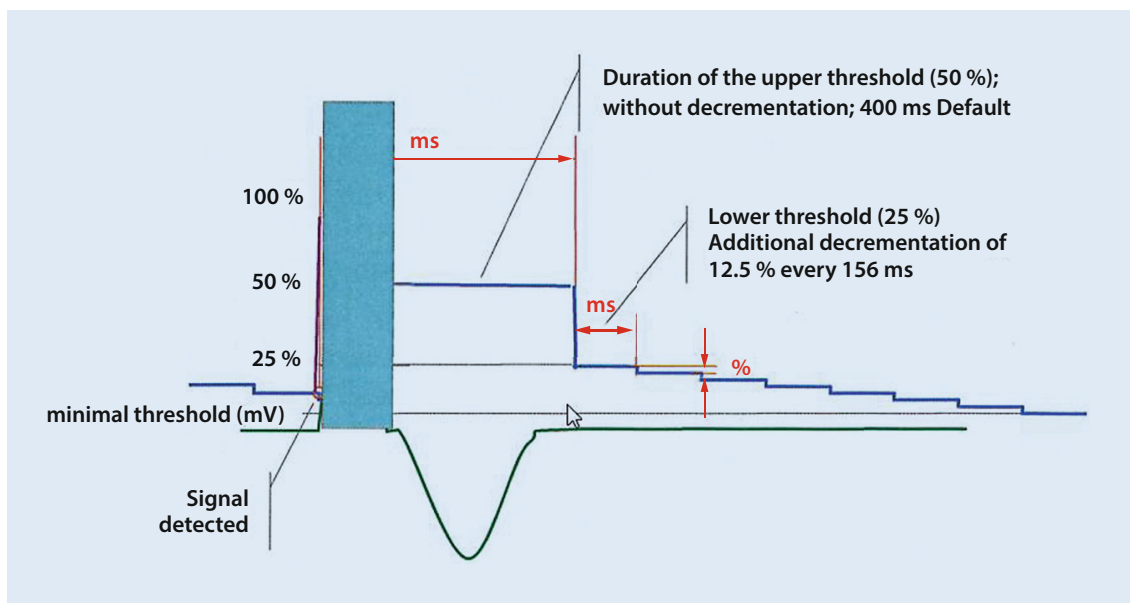

Fig. $2 \Delta$ Automatic sensitivity control with ventricular pacing 


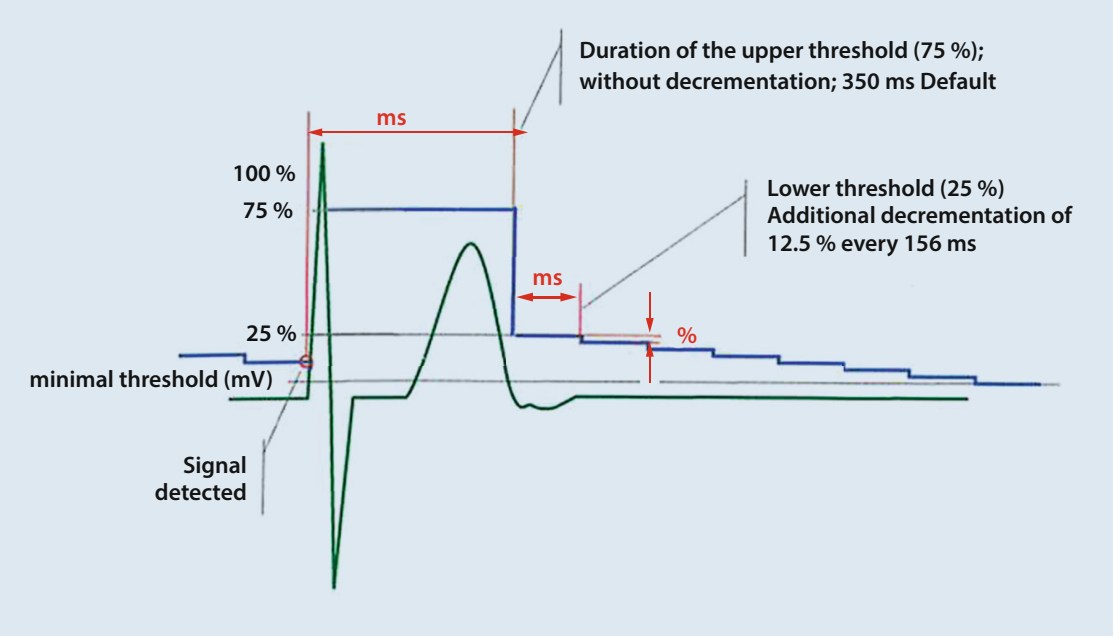

Fig. 3 Automatic sensitivity control with settings for enhanced T-wave suppression: the upper threshold is increased to $75 \%$ of the measured R-wave amplitude. Additionally, high-pass filtering is increased from 24 to $32 \mathrm{~Hz}$ to reduce low-frequency signal components

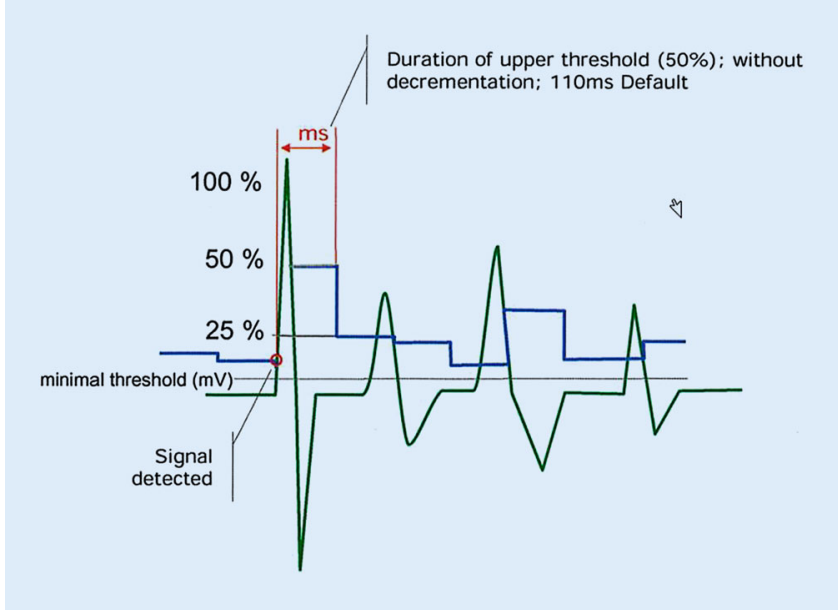

Fig. $4<$ Automatic sensitivity control with settings for enhanced VF sensitivity: the upper threshold duration after sense is decreased to $110 \mathrm{~ms}$. Additionally, high-pass filtering is increased from 24 to $32 \mathrm{~Hz}$

sensing and for a higher VF sensitivity. Modification of all sensing parameters (e.g., filter characteristics, threshold levels, holding periods, blanking periods, refractory periods) is possible with support from an experienced BIOTRONIK employee in case of unusual signals or in special troubleshooting situations.

\section{Ventricular sensitivity}

\section{Standard settings}

In the standard setting, the sensing stage is blanked for $110 \mathrm{~ms}$ after each sensed $\mathrm{R}$-wave. Each $\mathrm{R}$-wave is measured and used to set the upper and lower thresholds. The upper threshold after sensing is set to $50 \%$ of the peak R-wave amplitude. Following the upper threshold duration
Then the sensing threshold will drop to $25 \%$ of the event amplitude followed by further decrementation as described above (• Fig. 2). Alternatively, the sensing threshold start value at the end of the blanking period can be also programmed to a fixed sensing threshold value $(3 \mathrm{mV}$ by default). This feature may be used when the patient has small amplitude R-waves $(<4 \mathrm{mV})$ or large $\mathrm{T}$-waves in particular after ventricular pacing.

\section{Enhanced T-wave suppression}

The characteristics of the automatic sensitivity control can be modified for troubleshooting purposes in case of ventricular oversensing due to large repolarization signals as T-waves. Two parameters are automatically changed with programming of enhanced T-wave suppression (TWS): 1) The high-pass filtering is increased from 24 to $32 \mathrm{~Hz}$ to reduce low-frequency signal components such as repolarization signals and respiratory artifacts and 2) the upper threshold is increased to $75 \%$ of the measured R-wave amplitude (• Fig. 3). While TWS can eliminate T-wave oversensing, the setting should be used with caution if the R-wave signal amplitude during $\mathrm{T}$-wave oversensing is low (e. g., less than $4 \mathrm{mV}$ ). In general, VF detection should be retested when the sensitivity setting is reprogrammed.

\section{Enhanced VF sensitivity}

This feature was specifically designed to improve VF detection when the VF signal is small, which can lead to ventricular undersensing (i. e., signal drop-out) during VF detection. Two adjustments are made to the settings of automatic sensitivity control: 1) the upper threshold duration after sense is decreased to $110 \mathrm{~ms}$ (• Fig. 4), 2) the high pass filtering is changed from 24 to $32 \mathrm{~Hz}$. Both adjustments ensure that the threshold approaches the minimum threshold more quickly. If undersensing is still present, the minimum threshold may also need to be adjusted. These settings should be also used with caution, as oversensing of the intrinsic complex may occur. Detection of VF should be retested when the sensitivity setting is reprogrammed. 


\section{Atrial sensitivity in dual-chamber ICDs and CRT-D devices}

The atrial automatic sensitivity control works similar to the ventricular standard settings. Each $P$-wave is measured and used to define upper and lower atrial sensing thresholds. The upper threshold is set to $50 \%$ of the peak $P$-wave amplitude and is hold for $350 \mathrm{~ms}$ after atrial sensed and paced events. Then the atrial sensing threshold decreases to $25 \%$ and decays $12.5 \%$ every $156 \mathrm{~ms}$ until either the minimum atrial threshold is reached or until the next atrial event, sensed or paced, has occurred (- Fig. 5).

\section{Postventricular atrial refractory period}

Postventricular atrial refractory period (PVARP) is used to prevent pacemakermediated endless loop tachycardia (ELT) by not allowing atrial events to be tracked by the device. Please note that programming long PVARP values in combination with long AV delays might result in reduced 1:1 tracking by the device and can increase the risk of retrograde conduction. In some cases a retrograde conduction test can be of help before making any programming changes. Generally, PVARP should be programmed 15-20 ms longer than the measured retrograde conduction time. Programming PVARP too long could result in nontracking of antegrade atrial events. Default settings are $175 \mathrm{~ms}$ and $325 \mathrm{~ms}$ for PVARP after premature ventricular contraction (PVC). Auto PVARP is an option that will automatically adjust the PVARP value when ELT occurs. The devices have an algorithm to recognize and terminate ELT. Following an ELT, the device will extend PVARP by $50 \mathrm{~ms}$. After seven days of no ELT, the PVARP value will be reduced by $50 \mathrm{~ms}$ to a minimum value of $175 \mathrm{~ms}$. PVARP after PVC is a parameter that is used to prevent ELT following PVC events by adding a fixed value of $150 \mathrm{~ms}$ to the programmed PVARP for that PVC event. By default, this value is $375 \mathrm{~ms}$; the maximum value is $600 \mathrm{~ms}$.

Herzschr Elektrophys 2016 :27:171-185 DOI 10.1007/s00399-016-0449-z

(c) Springer-Verlag Berlin Heidelberg 2016

\section{T. Brüggemann · D. Dahlke · A. Chebbo · I. Neumann}

\section{Tachycardia detection in modern implantable cardioverter-defibrillators}

\section{Abstract}

Implantable cardioverter-defibrillators (ICD) have to reliably sense, detect, and treat malignant ventricular tachyarrhythmias. Inappropriate treatment of non lifethreatening tachyarrhythmias should be avoided. This article outlines the functionality of ICDs developed and manufactured by BIOTRONIK. Proper sensing is achieved by an automatic sensitivity control which can be individually tailored to solve special underand oversensing situations. The programming of detection zones for ventricular fibrillation (VF), ventricular tachycardia (VT), and zones to monitor other tachyarrhythmias is outlined. Dedicated single-chamber detection algorithms based on average heart rate, cycle length variability, sudden rate onset, and changes in QRS morphology as used in ICDs by BIOTRONIK are described in detail.
Preconditions and confirmation algorithms for therapy deliveries as antitachycardia pacing (ATP) and high energy shocks are explained. Finally, a detailed description of the dual-chamber detection algorithm SMART is given. It comprises additional detection criteria as stability of atrial intervals, 1:1 conduction, atrial-ventricular (AV) multiplicity, AV trend, and AV regularity to differentiate between ventricular and supraventricular tachyarrhythmias.

\section{Keywords}

BIOTRONIK · Implantable cardioverter defibrillator - Ventricular fibrillation - Ventricular tachycardia - Tachycardia discrimination . Single-chamber detection - Dual-chamber detection

\section{Erkennung von Tachykardien in modernen implantierbaren Kardioverter-Defibrillatoren}

\section{Zusammenfassung}

Ein implantierbarer Kardioverter-Defibrillator (ICD) muss in der Lage sein, maligne ventrikuläre Tachyarrhythmien zuverlässig zu erkennen, zu klassifizieren und zu behandeln. Eine unangebrachte Therapieabgabe bei nicht lebensbedrohlichen Tachyarrhythmien sollte vermieden werden. Im Rahmen dieses Artikels soll die Funktionsweise von ICDs der Firma BIOTRONIK dargelegt werden. Eine korrekte Erkennung von Herzaktionen wird durch eine automatische Wahrnehmungsfunktion erreicht, die auch die Möglichkeit einer individuellen Anpassung bietet, um spezielle Wahrnehmungsprobleme (z. B. Over- und Undersensing) zu lösen. Die Programmierung von Detektionszonen für die Erkennung von Kammerflimmern, Kammertachykardien und zur Überwachung anderer Tachyarrhythmien wird besprochen. Dezidierte 1-KammerDetektionsalgorithmen, wie sie in ICDs der Fa. BIOTRONIK verwendet werden, basieren auf der gemittelten Kammerfrequenz, der Variabilität der Kammerzykluslängen, dem
Vorliegen eines plötzlichen Anstiegs der Herzfrequenz und auf Veränderungen der QRS-Morphologie. Weiterhin werden Vorbedingungen und Bestätigungsalgorithmen für die Abgabe einer antitachykarden Stimulation bzw. eines hochenergetischen Schocks erläutert. Schließlich wird eine detaillierte Beschreibung des 2-Kammer Detektionsalgorithmus SMART von BIOTRONIK gegeben. Hier werden zusätzliche Detektionskriterien wie die Vorhoffrequenz, die Variabilität der Vorhofzykluslängen, 1:1-AV/VA-Überleitung, atrioventrikuläre (AV) Multiplizität, AV-Trend sowie die Regelmäßigkeit der AV-Überleitung verwendet, um zuverlässig zwischen ventrikulären und supraventrikulären Tachyarrhythmien zu unterscheiden.

\section{Schlüsselwörter}

BIOTRONIK · Implantierbarer Defibrillator . Kammerflimmern · Kammertachykardie . Tachykardiediskriminierung · 1-KammerDetektion · 2-Kammer-Detektion 


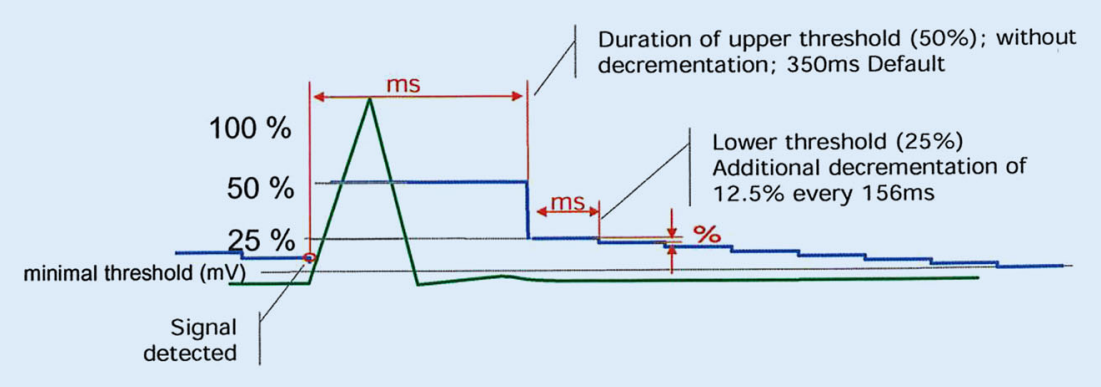

Fig. 5 A Automatic sensitivity control in the atrium

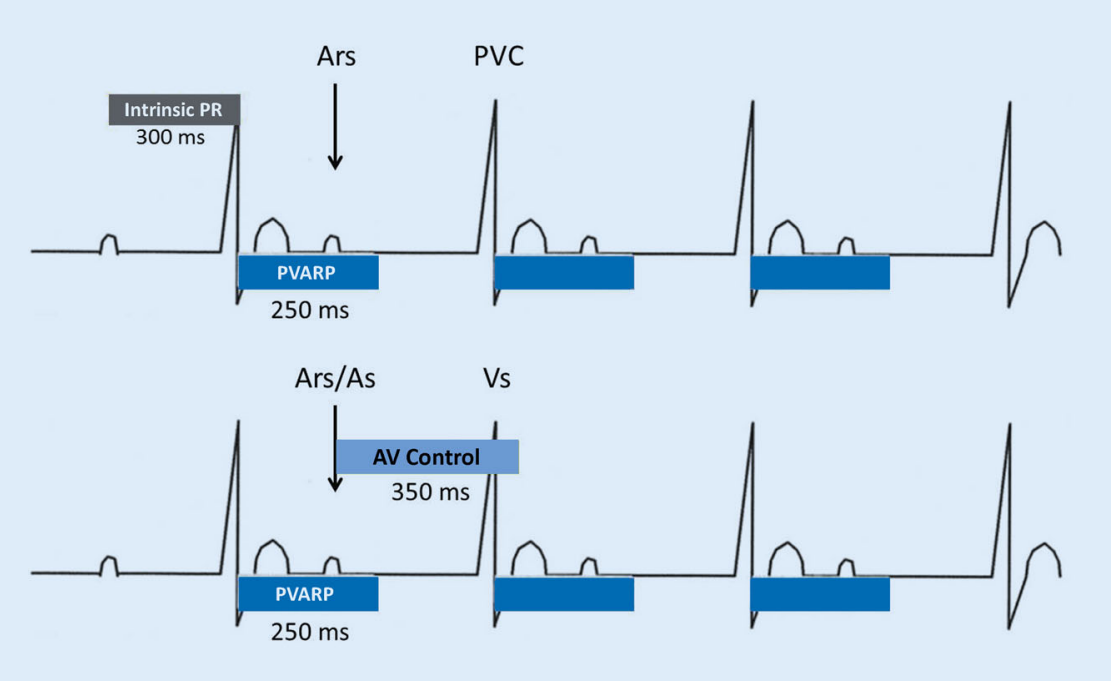

Fig. $6 \Delta$ The atrial events are seen within the postventricular atrial refractory period (PVARP) and are considered to be atrial refractory-sensed event (Ars) events. The subsequent ventricular event is classified as a premature ventricular contraction (PVC). Ars events do not start the lower rate timer. However, as the Ars event is within $350 \mathrm{~ms}$ of the subsequent ventricular event, discrimination as atrial sensing (As) will count those events as AsVs events in the statistics

\section{Far-field protection}

This feature is activated in the standard program to prevent oversensing of crosstalk events to avoid inappropriate therapies or mode switching. Farfield protection means that the signals that occur during this time interval are not sensed by the device (i. e., blanking period). As a result, there will be no annotations on the marker channel, and these events are not recorded in the device diagnostics.

Far-field protection after a ventricular-sensed event (Vs) initiates an atrial blanking period before and after each ventricular-sensed event. The blanking period before Vs is fixed to $16 \mathrm{~ms}$, the total duration of this blanking period is programmable (default $75 \mathrm{~ms}$ ). Far-field protection after ventricular-paced events
Discrimination after atrial sensing (As)

This feature has been introduced to provide a more accurate classification of premature ventricular contraction (PVC) and event timing. If an RVsensed event occurs within $350 \mathrm{~ms}$ after an atrial refractory-sense event (Ars), the device assumes the Vs event to be a response to the previous atrial (Ars) event (i. e., AV conducted). As a result, the device will consider the Vs event to be an AsVs event, rather than an Ars event followed by a PVC, and will be annotated as such. This will provide a more accurate PVC count and event timing (• Fig. 6).

\section{Detection}

To ensure that appropriate ICD therapy is delivered, ventricular tachycardia (VT) and ventricular fibrillation (VF) must be distinguished from normal sinus rhythm (SR) and supraventricular tachycardias (SVT). This is accomplished by proper device sensing, as well as appropriate detection. The ICD detects and measures the rate of sensed cardiac signals to discriminate ventricular tachyarrhythmias from SVTs, SR or sinus bradycardia. This is accomplished through programmable rate detection parameters in the device. When a tachyarrhythmia is present, the ICD classifies the arrhythmia and delivers appropriate therapy. In case of a spontaneous termination of the tachyarrhythmia during detection, the counters are reset in case of five consecutive intervals that do not fall into any tachyarrhythmia zone with therapy (so-called "short termination"). If a tachyarrhythmia continues following the first therapy attempt, then the ICD will redetect the tachyarrhythmia and deliver subsequent therapies as necessary.

\section{Ventricular tachyarrhythmia detection}

Classification of cardiac signals is accomplished primarily by measuring cardiac cycle lengths (R-R, P-R, and P-P). In addition, the ICD can also utilize abrupt changes in rate, irregularity of the cycle 

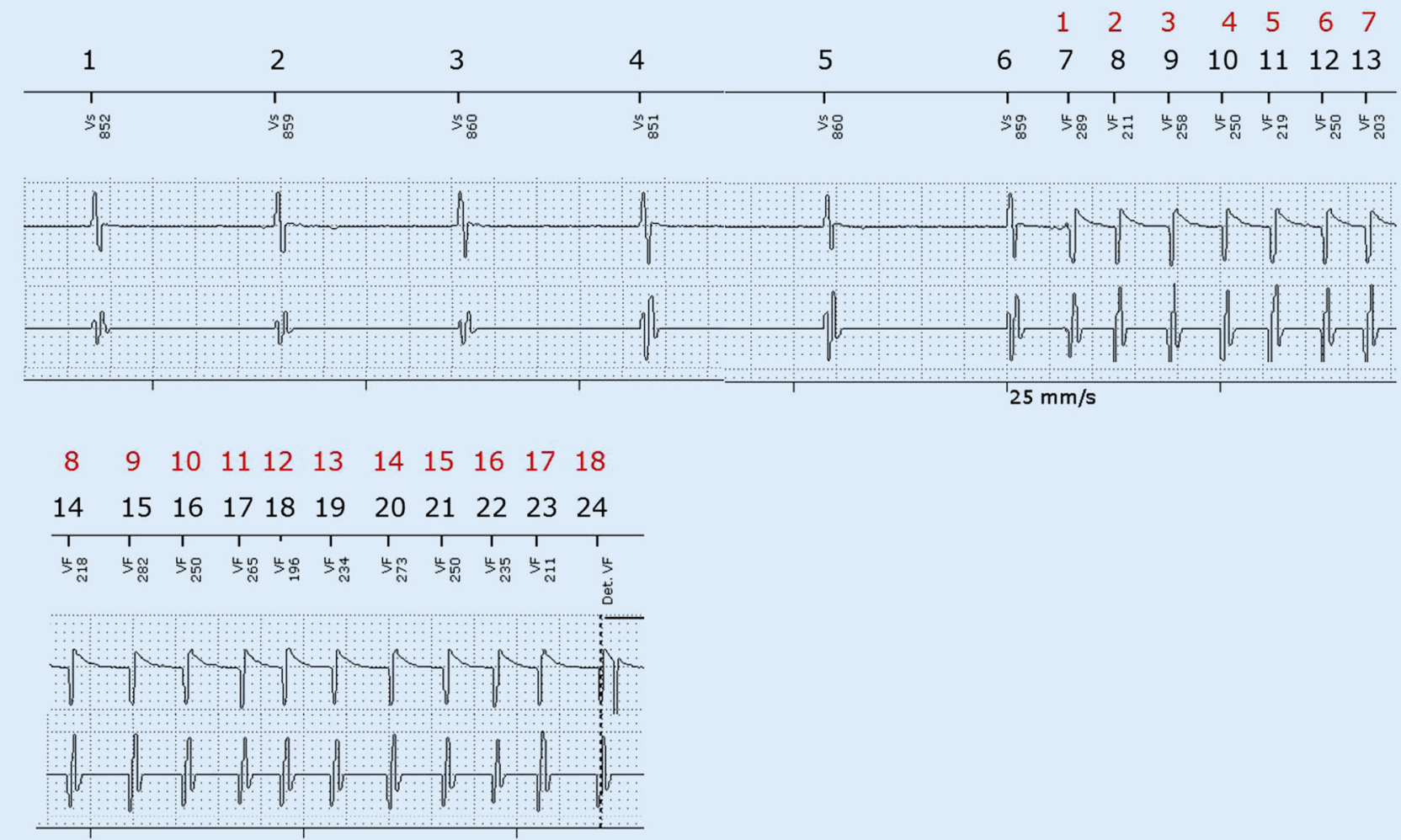

Fig. $7 \Delta$ Example of VF detection. The top line indicates the detection markers with the related cycle length (Vs ventricular sense, VF ventricular fibrillation), the second line shows the electrogram (EGM) signal recorded between the shock coil and the ICD housing (i. e., far-field EGM), and the lowest line the signal recorded between the tip and the ring electrode of the ICD lead (i. e, near-field EGM). The VF zone is programmed to 18 out of 24 events at an interval shorter $\leq 300 \mathrm{~ms}$, or a ventricular rate $\geq 200 \mathrm{bpm}$. A sliding window of 24 intervals (blacknumbers) is continuously monitored for VF intervals (rednumbers). VF is detected (Det. VF) when 18 out 24 intervals are found to be $300 \mathrm{~ms}$ or less (vertical dotted line). A horizontal bar below the "Det. VF" marker indicates that the capacitors are beginning to be charged for VF treatment

lengths and other criteria to further differentiate ventricular tachyarrhythmias. Each detected ventricular tachyarrhythmia is classified into one of the following zones:

- VT-1 lower rate ventricular tachycardia,

- VT-2 higher rate ventricular tachycardia, or

- VF ventricular fibrillation.

Each zone is programmable to a separate rate with the zone limit defining the lowest rate in each zone. The upper rate limit of each zone is equal to the zone limit of the next higher class, creating a continuous range of rate classes.

\section{VF detection}

Detection in the VF zone relies on an $\mathrm{X}$ out of $\mathrm{Y}$ detection criterion. If $\mathrm{X}$ intervals within a rolling window of $Y$ inter- vals are shorter (i. e., the rate is faster) than the VF detection interval/rate limit, then VF is declared as detected (• Fig. 7) and VF therapy will be delivered. Recommended combinations are 30 out of 40,24 out of 30 or shorter combinations (e. g., 18 out of 24). The rate limit should be programmed between $211 \mathrm{bpm}$ and $231 \mathrm{bpm}$ (i. e., the R-R interval between $284 \mathrm{~ms}$ and $260 \mathrm{~ms}$ ) [1]. VF redetection counters can be programmed differently from initial VF detection criteria in newer generation devices.

\section{Ventricular-only detection for VT}

When a VT zone is programmed, the ventricular-only detection algorithm uses a programmable VT interval count and based on a rate/interval cut-off criteria. This means that any time the rhythm crosses the programmed VT detection interval, the ICD will classify the event as VT. VT detection relies on simple up/ down counters (i.e., separate for VT1 and VT2 zone), ventricular rate, and single-chamber enhancement. Detection enhancements such as Onset, Stability and MorphMatch can be added to help to discriminate VT from SVT. These detection enhancements are sometimes referred to as "therapy inhibitors", as both interval count and the programmed detection enhancement criteria must be met to classify an arrhythmia. The VT detection counter determines the minimal number of VT events for each zone that must be counted to initiate VT detection and therapy delivery. The redetection counter of the VT1 zone must be greater than the redetection counter of VT2 zone and the "X" value of the VF zone detection counter. 


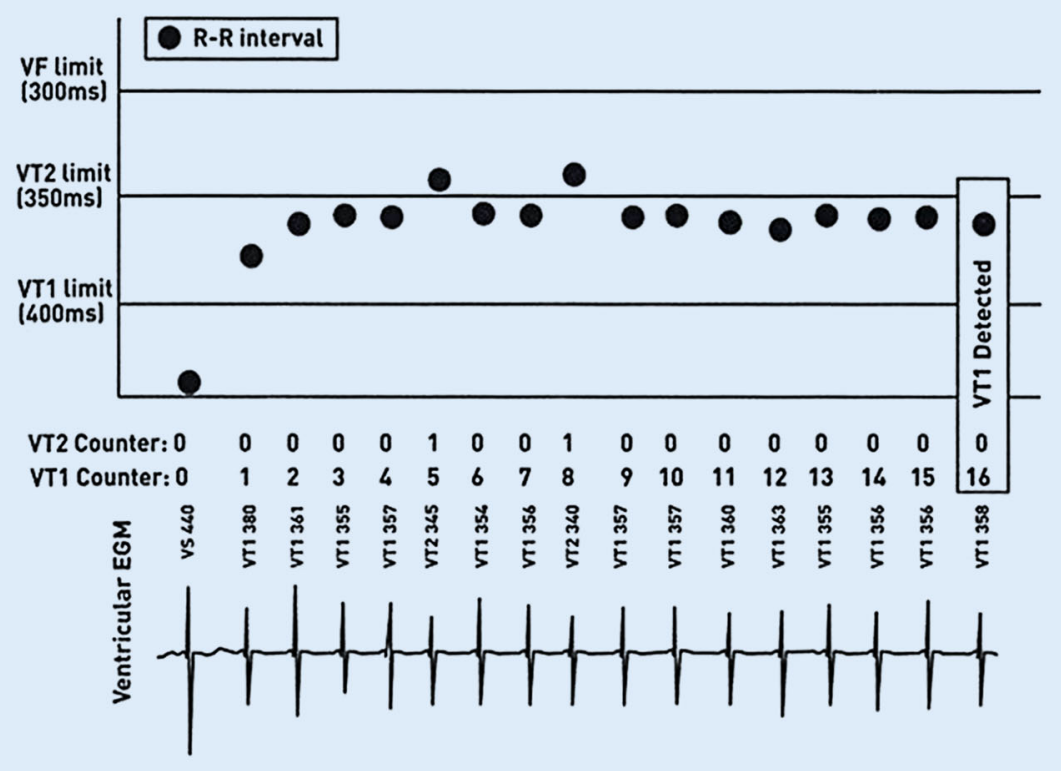

Fig. $8 \Delta$ Example for rate-only VT1 detection. In this case, there are three tachyarrhythmia zones programmed (i. e., VT1, VT2, VF). VT detection begins with the first short interval that falls into the VT1 zone. The VT1 detection counter increases with each interval shorter than the VT1 limit of $400 \mathrm{~ms}$, including intervals that fall into the VT2 zone. The VT2 detection counter increases with each interval shorter than the VT2 limit of $350 \mathrm{~ms}$ and decrements with each interval longer than the VT2 limit.VT1 detection is declared and therapy delivered when the VT1 detection counter reaches the programmed value of sixteen

Rate only. When Rate Only is used, detection is met when the programmed number of intervals exceed the programmed detection count. Each time an interval is shorter than the programmed VT zone limit, the VT counter increments (+1). Conversely, the VT counters decrement by one $(-1)$ when an interval is longer than the programmed limit for that zone. All intervals in the VT2 zone will also increment the VT1 counter (• Fig. 8). Intervals longer than the defined detection zones decrement the counter for that zone.

Stability. Stability is a detection enhancement to assist in determining if a tachyarrhythmia is VT or atrial fibrillation with a rapid ventricular response. Stability refers to the stability of R-R intervals. Monomorphic VT demonstrates a stable R-R interval, while in atrial fibrillation the R-R intervals are typically unstable. Stability is evaluated on a beat-tobeat basis. Stability is used for detection and redetection. The Stability criterion is satisfied for a given ventricular interval when the difference between the current interval and each of the three preceding intervals is less than the programmed Stability limit. Unstable events reset the VT counters to zero and can potentially delay therapy. Programming a higher value of stability (i. e., allowing less stable intervals to be classified stable) can result in therapy delivery for atrial fibrillation, while programming a lower value may result in inhibition of therapy for VTs which may be slightly unstable (• Fig. 9). The recommended default setting for single-chamber ICDs is $40 \mathrm{~ms}$ or $12 \%$ for newer generation ICDs [1].

Sudden onset. Sudden Onset is a detection enhancement that can help to determine whether a tachyarrhythmia is of ventricular or supraventricular origin. Spontaneous VTs typically demonstrate a sudden increase in ventricular rate, while sinus tachycardias increase the ventricular rate slowly over time (• Fig. 10). Sudden onset is programmed to a default value of $20 \%$ and is available in both VT1 and VT2 zones. This Onset algorithm is designed in order to prevent single fast events as extra beats to fulfill Onset and potentially lead to inappropriate therapy. Onset applies to the ventricular chamber only. Once Onset is fulfilled, it is declared for the entire episode, including redetection. It can be reset by five consecutive intervals that are out of any VT/VF zones with therapy.

Morphology. An additional detection enhancement based on changes in the QRS morphology is available when using ventricular-only detection for VT. The QRS morphology is analyzed in a $250 \mathrm{~ms}$ window around the peak of QRS in the farfield EGM. This MorphMatch algorithm is based on beat-to-beat analysis of the QRS peak amplitude, the QRS area, and four major QRS deflections. The difference to the reference is calculated for every single beat after alignment of the actual beat to the reference QRS. Normal beats continuously update the reference QRS and an average variability of normal beats is calculated. This average variability (i. e., mean difference to the reference) plus a programmable safety margin result in a morphology threshold to declare a QRS complex as abnormal (e. g., VT) or normal (e.g., SVT) (• Fig. 11). The MorphMatch criterion can be programmed active or inactive.

In parallel to the VT counter a MorphMatch counter increments if the QRS morphology is classified as abnormal. With MorphMatch active an episode is declared as VT when the VT detection count is met, stability and onset are fulfilled, and at least $50 \%$ of the QRS complexes have an abnormal morphology according to MorphMatch. Ifless than $50 \%$ of the QRS complexes have an abnormal morphology according to MorphMatch this episode is declared as SVT (• Fig. 12).

\section{Detection criteria associated with therapy delivery}

ATP one-shot. ATP One-shot is a feature that delivers a single ATP therapy for a stable rhythm in the VF zone prior to shock charging. The rhythm in the VF zone must be regular (i.e., within $12 \%$ stability), such as a stable fast monomorphic VT. There is no rate limit for ATP Oneshot. Once the ATP is delivered, charging of the capacitors begins automatically. During charging, the device continues 


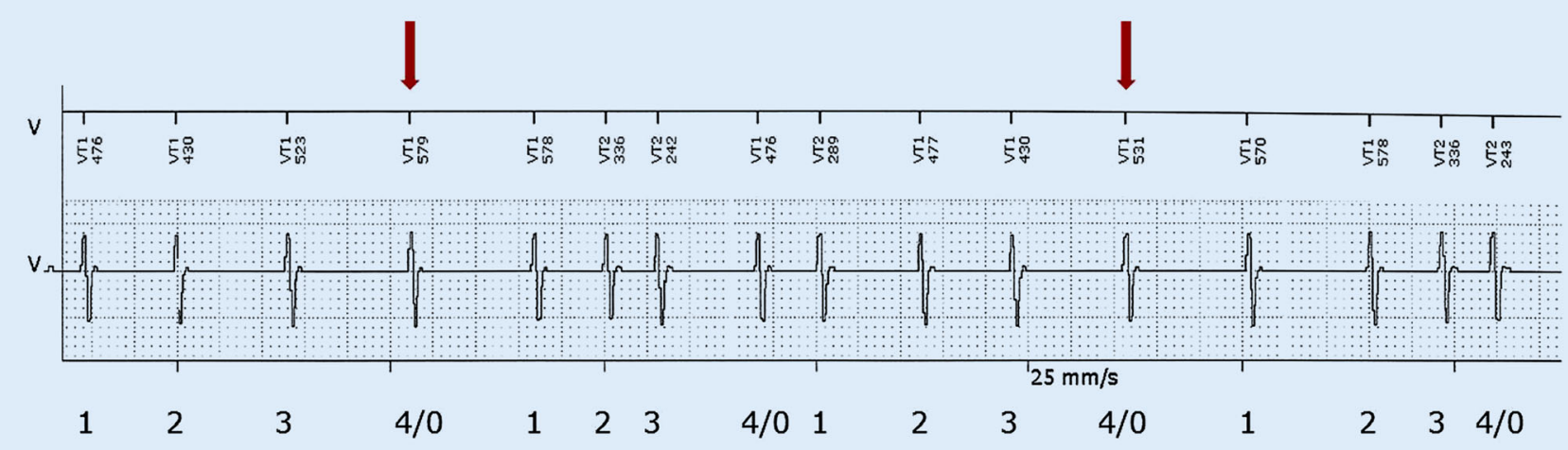

Current interval $=579 \mathrm{~ms}$

Compare to three previous intervals

$579-523=56 \mathrm{~ms}$ ( $>40 \mathrm{~ms})$ and

$579-430=149 \mathrm{~ms}(>40 \mathrm{~ms})$ and

$579-476=103 \mathrm{~ms}$ (>40 ms)

Stability not met
Current interval $=531 \mathrm{~ms}$

Compare to three previous intervals

$531-430=101 \mathrm{~ms}$ (>40 ms) and

$531-477=54 \mathrm{~ms}(>40 \mathrm{~ms})$ and

$531-289=242 \mathrm{~ms}$ (>40 ms)

Stability not met

Fig. $9 \Delta$ Calculation of Stability. If the VT1 counter reaches four, the stability check is performed by comparing the current interval length with the previous three intervals. If the result of one comparison does not fulfill the stability criterion, the VT counter is reset to zero. Recommended values are $40 \mathrm{~ms}$

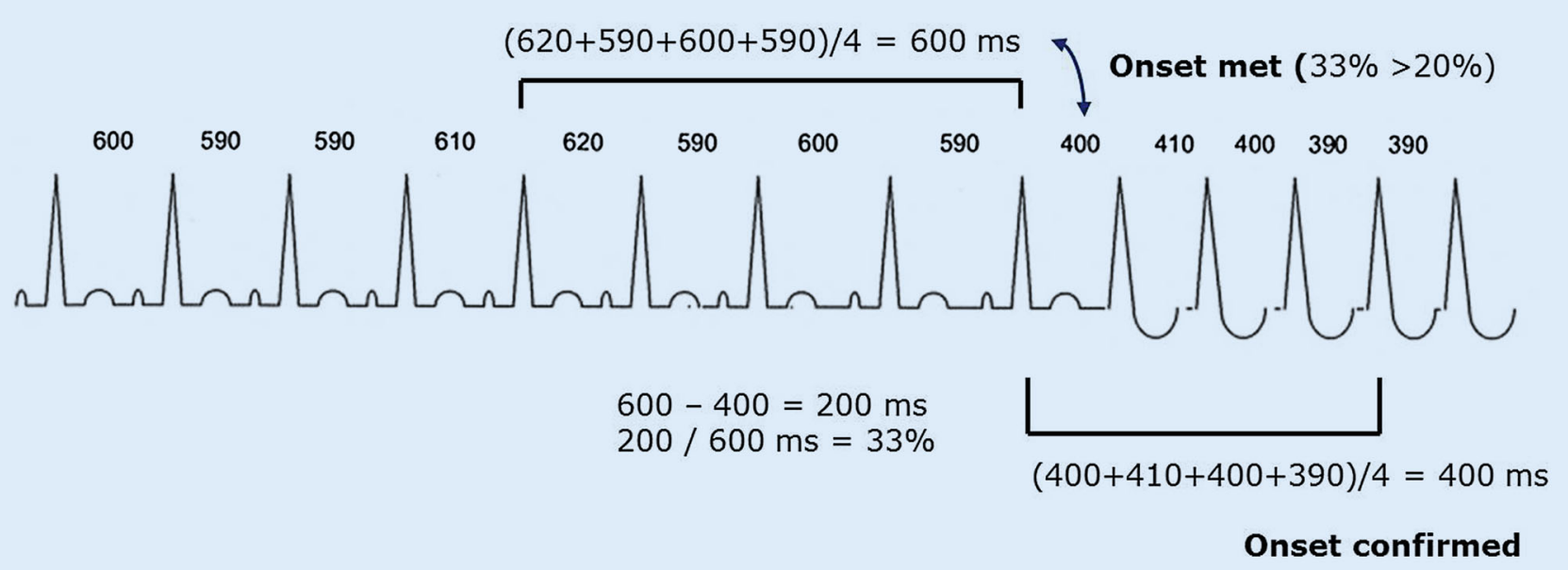

Fig. $10 \Delta$ Calculation of Onset. Once a short ventricular interval (i. e., $400 \mathrm{~ms}$ ) in a VT/VF zone meets the onset criteria in comparison to the previous 4 -interval average interval (i.e., $600 \mathrm{~ms}$ ), the device uses that interval and the next three additional intervals to determine a new 4-interval average. This average is compared to the previous 4-interval average before the onset interval. Onset is confirmed when the difference between the current 4-interval average and the previous 4-interval average exceeds the programmed limit (20\%). The Onset criteria are fulfilled when (1) onset is met by a single interval and (2) confirmed by the following three intervals 


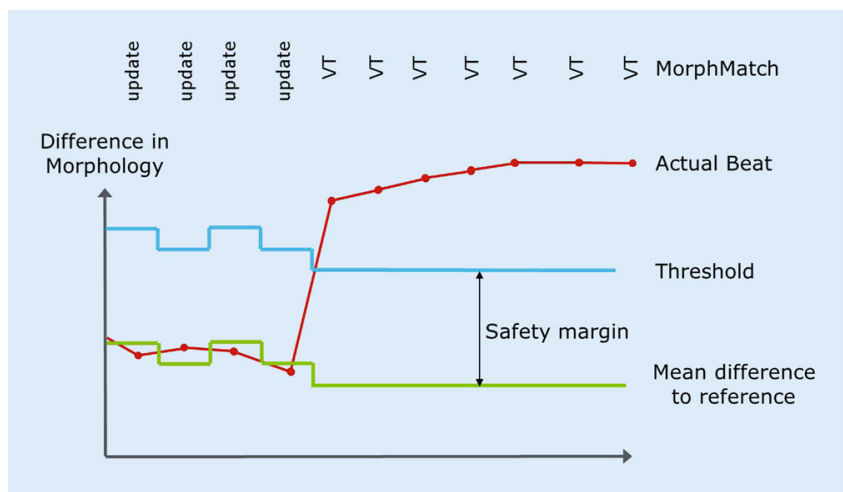

Fig. $11 \Delta$ This diagram illustrates the functionality of the MorphMatch algorithm following the onset of a VT. Up to the first beat of the VT, normal beats update the mean difference to the reference (green line) and the morphology threshold (blue line). With the onset of the VT, the actual differences exceed the morphology threshold (red line) and the MorphMatch counter starts to increase. The mean difference to the reference and the morphology threshold are frozen during an ongoing VT

to classify the rhythm and charging is aborted once three long intervals (out of four) occur (- Fig. 13). ATP One-shot is automatically disabled after four consecutive unsuccessful attempts. When the device is interrogated, ATP One-shot will automatically re-enabled. After analyzing the episodes, the user can decide to deactivate this therapy option.

Shocks with confirmation ("non-committed shocks"). Rhythm classification continues through the charging period when shock confirmation is programmed to ON. If the device detects during charge three out of four slow events (i. e., sinus or paced), then the device aborts shock therapy ( $\bullet$ Fig. 14). If self-termination occurs or no further VT/VF intervals are available to synchronize the shock, then the shock delivery will be withheld. The device then begins the redetection/ termination process. The aborted shock energy is slowly released into an internal resistor and may make up to $10 \mathrm{~min}$ to bleed off. If charging ends, a $70 \mathrm{~ms}$ blanking interval is started to avoid oversensing of artefacts due to the switch of high voltage. The shock energy is delivered $30 \mathrm{~ms}$ after the next VT/VF interval (• Fig. 15).

Confirmation applies to all shocks within the programmed VT or VF zone. With confirmation programmed $\mathrm{ON}$, the first shock in the detected ventricular tachyarrhythmia zone is non-commit- ted. Each time a shock is aborted, the next shock after redetection is automatically committed. The device will not allow two aborted shocks in a row to occur. This is a safety feature, should intermittent undersensing of a ventricular tachyarrhythmia be present, to prevent therapy inhibition.

Shocks without confirmation ("committed shocks"). A committed shock is a shock delivered after (re-)detection and charge. There is no attempt to recheck or verify the rhythm prior to shock delivery. The device tries to synchronize with the first ventricular sensed or paced event (• Fig. 16). However, if synchronization is not possible, the device delivers the shock asynchronously within two seconds.

Detection of the end of episode (termination detection). Termination of a ventricular tachyarrhythmia episode is based on a nonprogrammable 12 out of 16 termination criterion. Termination of an episode is declared when twelve out of a sliding window of 16 intervals are longer (i. e., the rate is slower) than the lowest programmed cutoff interval/rate limit with therapy. This includes also postshock and antibradycardia pacing intervals. If 12 long intervals are measured before the first window of 16 intervals, termination is declared with the twelfth interval (• Fig. 13). If detection starts anew following termination, all programmed therapy is available for treatment.

\section{Criteria used for dual-chamber AV discrimination (SMART)}

When the SMART algorithm for dualchamber detection is active, information from the atrial and ventricular chambers of the heart is used for VT classification. The algorithm is specifically designed to detect VT while withholding therapy upon detection of an SVT. The algorithm performs classification on a beat-to-beat basis. Classification begins after the cycle length is measured and determined to lie within the programmed VT zones. Thereafter, a series of tests is performed to determine if the arrhythmia is atrial or ventricular in origin. SMART detection is programmed ON by default in dual-chamber and CRTICDs when a VT zone is programmed. It is not available in the VF zone.

\section{Rate (interval)}

SMART Detection uses a sliding average of atrial and ventricular intervals to compare the overall atrial and ventricular rates (- Fig. 17). This is used to determine whether the rates in the atrium and in the ventricle differ from each other. This is the first criterion of the SMART Detection algorithm.

\section{Stability}

With SMART Detection on, stability is checked on a beat-to-beat basis in the atrium and the ventricle (- Fig. 18). Although the calculation of stability is determined independently in both chambers, the same default of $12 \%$ is used for each chamber.

\section{Sudden onset}

Sudden Onset is used in the SMART Detection algorithm when the atrial and ventricular rhythms are stable and equal in rate to distinguish VT from slow onset sinus tachycardia. Sudden Onset is applied in the ventricular chamber only and programmed at $20 \%$ by default (๑ Fig. 10). 


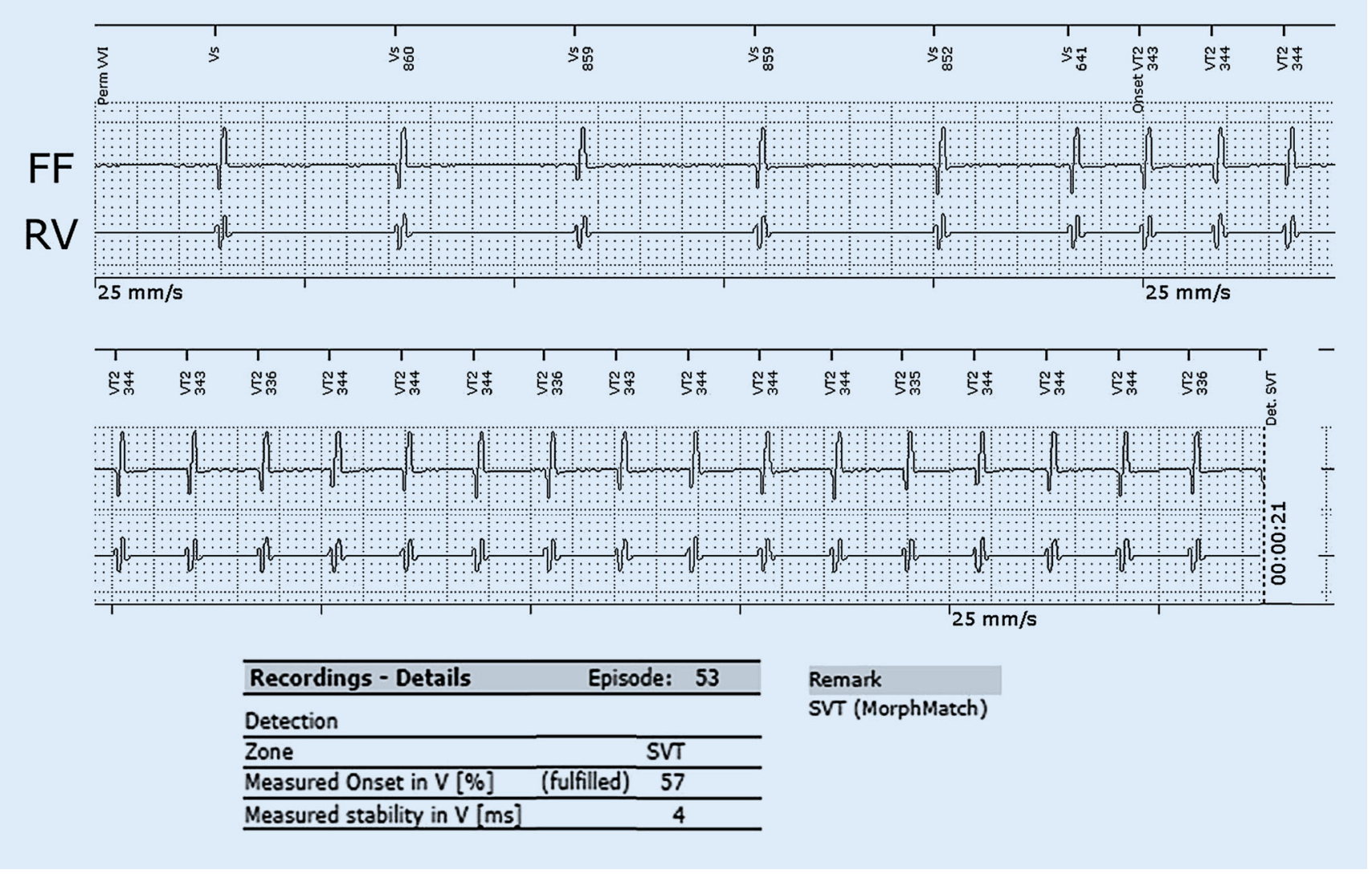

Fig. 12 A Tracing from an episode with detection in VT2 zone. The top line indicates the marker channel, the second line shows the far-field EGM (FF) from RV coil to ICD can, and the lower line shows the near-field RV EGM recorded between RV tip and RV ring (RV). Onset and Stability are fulfilled with $57 \%$ and $4 \mathrm{~ms}$, respectively (see marker and recording details). A detection in VT2 zone is achieved by 20 consecutive VT2 intervals. However, MorphMatch is active and overrules the VT detection by declaring the episode as an SVT. This decision seems to be correct due to very little changes in morphology in the far-field EGM

\section{AV trend}

SMART Detection measures the AV trend to determine whether a consistent pattern between the atrial and ventricular rhythms (AV association) exists. This is performed when the atrial and ventricular rates are stable and equal. With each new ventricular interval, the algorithm checks to see whether the four most recently measured AV intervals are in strict increasing or decreasing order (• Fig. 19). This strict order of AV intervals defines a consistent trend towards $\mathrm{AV}$ shortening or $\mathrm{AV}$ prolongation that is indicative of $\mathrm{AV}$ dissociation.

\section{AV regularity}

SMART Detection measures AV regularity to determine whether a consistent pattern between the atrial and ventricular rhythms is present. The device compares the current AV interval against the three previous AV intervals without av- eraging. An AV interval is determined to be regular if the interval does not differ from the three preceding AV intervals by more than the AV limit. The AV limit is a predetermined value equal to half of the stability limit, or $6 \%$, by default.

\section{AV multiplicity}

To determine whether atrial flutter is present, SMART Detection looks for a consistent $N: 1$ pattern (e.g., 2:1, 3:1). An $N: 1$ pattern, called Multiplicity, is confirmed when the average ventricular interval is found to be a multiple of the average atrial interval with a tolerance of $\pm 12 \mathrm{~ms}$ to allow for minor normal variances. Multiplicity, like all SMART Detection criteria, is evaluated on a beatto-beat basis (- Fig. 20).

\section{Dual-chamber AV discrimination algorithm (SMART)}

SMART Detection is a very sophisticated detection algorithm for discrimination of VT and SVT. It is designed to increase the detection specificity (i. e., to increase the likelihood that atrial tachycardia is not declared a VT) without sacrificing sensitivity (i. e., the ability to detect and treat all clinical VTs of the patient). This can help to avoid inappropriate therapies. With SMART Detection, inappropriate shocks for SVT are minimized without compromising accurate VT detection and therapy.

Each time an interval is found to lie within one of the programmed VT zones, SMART Detection follows a series of decisions to determine whether the interval meets criteria for a VT count or an SVT count. VT criteria affect VT counters, while SVT intervals affect an SVT 

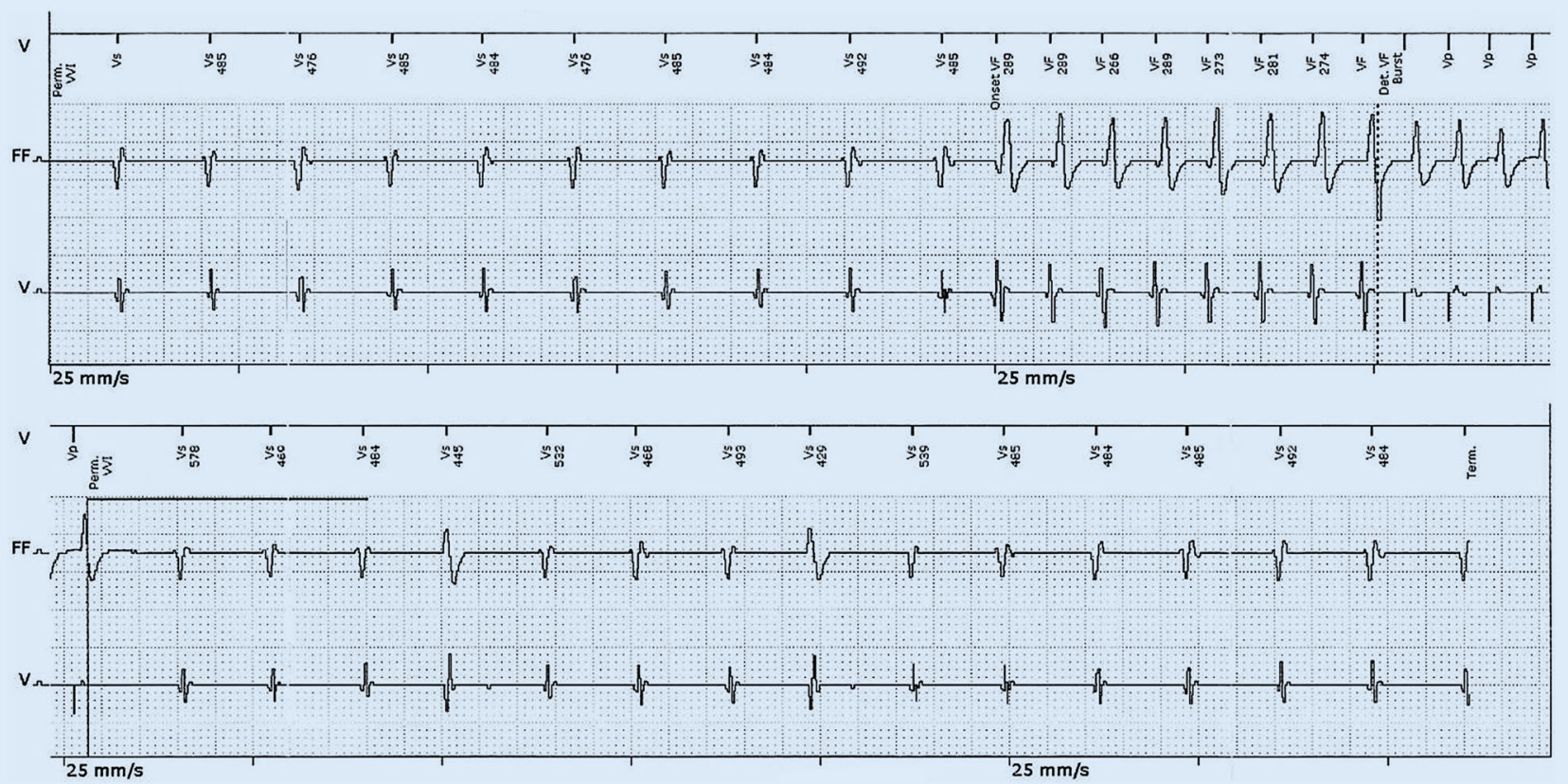

Fig. 13 - Example of a fast, stable VT detected in the VF zone. The VF detection is programmed to 8 out of 12 VF intervals. The dotted line indicates that the VF detection criterion is fulfilled (Det.VF). An ATP-one-shot (a single ATP attempt in the VF zone) is delivered because the last four intervals prior to VF detection were stable within a $12 \%$ tolerance limit. ATP one-shot is a burst of five ventricular pulses with a coupling interval of $88 \%$ (Burst). Immediately after ATP delivery the charging of the capacitors is started (black horizontal line). Capacitor charging stops at the end of the third ventricular sense (Vs) interval due to the confirmation algorithm (three out of four intervals outside VT/VF zones with therapy programmed). The termination (Term.) of the episode is declared as soon as 12 out of 16 intervals are outside VT/VF zones with therapy.Perm. VVI permanent pacing is programmed VVI, FF Far-field IEGM recorded between distal coil and can, Onset sudden onset criterion fulfilled, $V$ ventricular IEGM recorded between RV-tip and RV-ring electrode

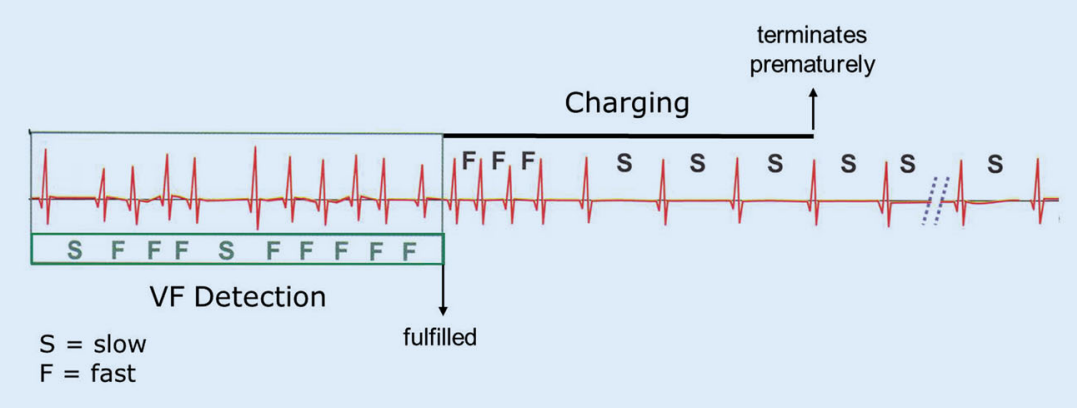

Fig. $14 \Delta$ Example of a fast ventricular tachyarrhythmia detected in the VF zone (8 out of 12 intervals are fast). ATP one-shot is not given due to instability (i.e., >12\%). Capacitor charging is stopped because three out of four intervals are slower and outside any VT/VF zone with therapy. This behavior is related to the confirmation algorithm switched ON and can be described as a "non-committed shock"

counter. VT intervals are labeled with VT1 or VT2 marker. SVT intervals are marked by one of four labels: AFib for atrial fibrillation, AFlut for atrial flutter, SinusT for sinus tachycardia, or 1:1 for atrial tachycardia.

Most tachyarrhythmias will occur when the ventricular rates are not equal to the atrial rates. Monomorphic VT without retrograde conduction, atrial flutter, and atrial fibrillation are identified by these portions of the decision tree (• Fig. 21).

The SMART Detection algorithm is also available in a single lead BIOTRONIK ICD system. This so-called DX system consists of a single coil ICD lead with an active fixation mechanism and floating atrial ring electrodes for atrial IEGM recording. A dedicated atrial sensing amplifier with a special filter characteristic allows recording of an atrial signal, good enough to operate the SMART dual-chamber detection in a dedicated DX ICD.

\section{VT counters with SMART Detection}

When the VT criteria are met and the interval lies in the VT1 zone, the VT1 counter increments by one (the VT2 counter is unaffected). When the VT criteria are met and the interval lies in the VT2 zone, the VT2 and the VT1 counter increments by one (so called "combined counting"). VF intervals do not increase the VT counters.

SVT intervals decrement the VT counters: Atrial Flutter by one, Atrial Fibrillation by four, Sinus Tachycardia by $1 / 4$ for initial and redetection. In addition, 1:1 tachycardias decrement the VT counters by $1 / 4$ for initial and $3 / 4$ for redetection. An SVT is declared when 


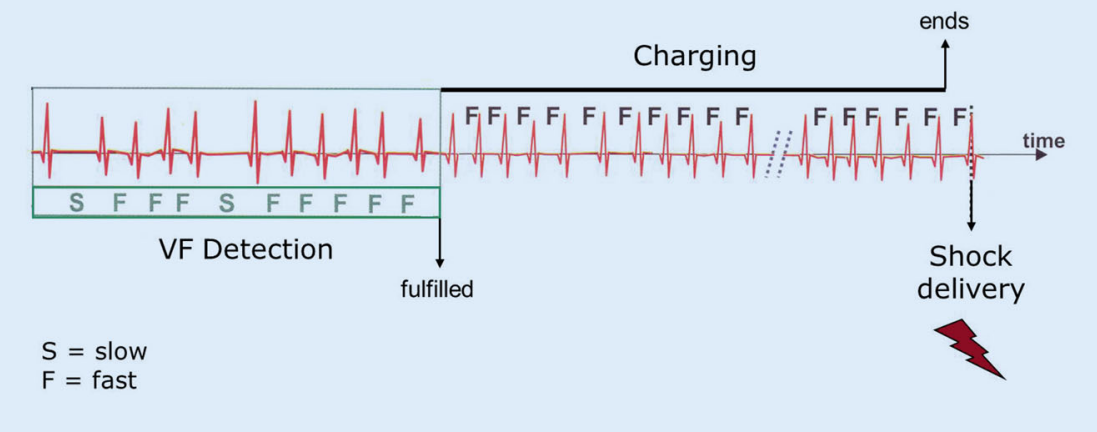

Fig. 15 - Example of a fast ventricular tachyarrhythmia detected in the VF zone (8 out of 12 intervals are fast). ATP one-shot is not given due to instability (i.e., $>12 \%$ ). Capacitors are fully charged because all intervals during charging are falling into a VT/VF zone with therapy. After charge end, the shockis delivered to the next fast VT/VF interval from a therapy zone. This behavior is related to the programmed confirmation algorithm and is called a "non-committed shock"

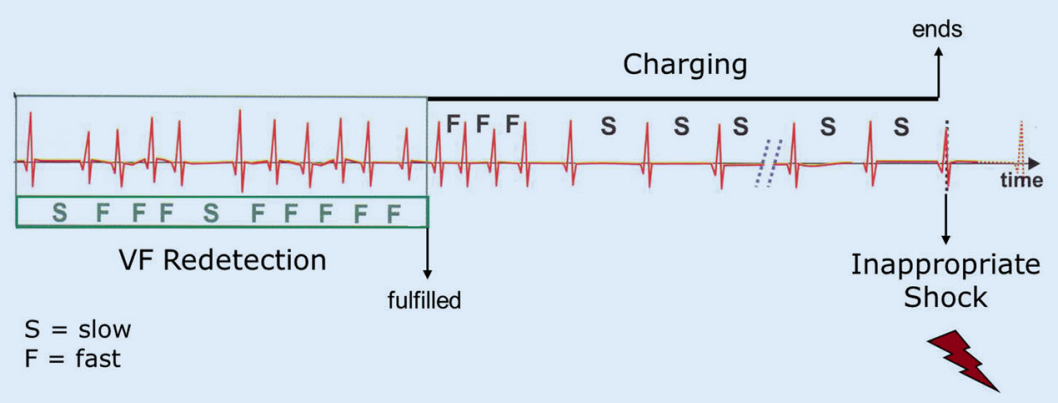

Fig. $16 \Delta$ Example of a fast ventricular tachyarrhythmia redetected in the VF zone after an unsuccessful first shock delivery. ATP-one-shot is not available after redetection. Capacitors are fully charged despite a spontaneous termination of the ventricular tachyarrhythmia with slow intervals, because the confirmation algorithm is switched OFF. After charge end, the shock is delivered to the next interval. This synchronization interval can be also a normal sinus interval. This therapy is called a "committed shock" and is an inappropriate shock in this example

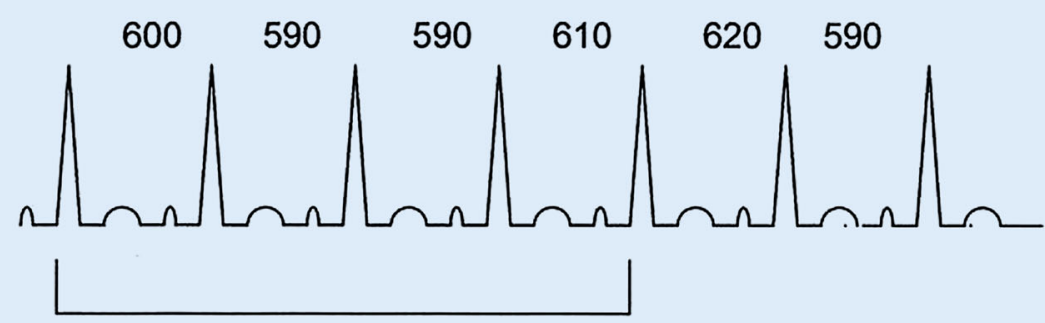

V sliding average $=(600+590+590+610) / 4=598$

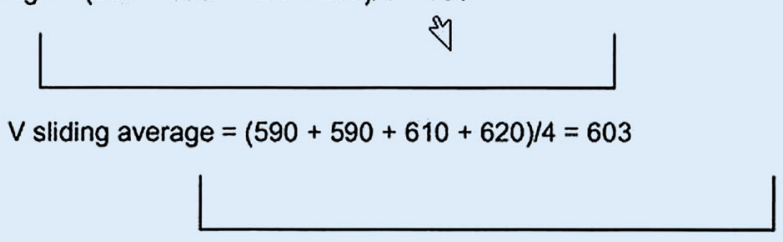

$V$ sliding average $=(590+610+620+590) / 4=603$

Fig. 17 \ Calculation of sliding averages forventricularcycle lengths. The sliding average is calculated using the four most recently measured intervals. With each new interval, the average is recalculated, deleting the oldest interval from the average and incorporating the most recently measured interval. In this way, the device has a window over time to assess ventricular as well as atrial rates the SVT criteria is met. The detection criteria for SVT is twice the VT zone count. For example, if the counter for a VT zone is programmed to 16 intervals, it will take 32 SVT intervals for an SVT episode to be declared (• Fig. 22).

\section{Detection of monomorphic/ polymorphic VT}

When a VT occurs without accompanying atrial tachyarrhythmia or without retrograde conduction $\mathrm{AV}$ discrimination is based on a comparison of rates alone-the ventricular rate is faster than the atrial rate. A monomorphic VT has a slow atrial rate, an accelerated ventricular rate, a stable QRS morphology, and stable R-R intervals. Polymorphic VT has a slow atrial rate, an accelerated ventricular rate, a varying QRS morphology, and instable R-R intervals. In both cases the single criterion ventricular rate faster than atrial rate will fulfill VT detection and subsequent therapy delivery (- Fig. 21, branch 1).

\section{Discrimination of atrial flutter}

Discrimination algorithms have difficulties to identify VT when it occurs in the presence of an ongoing atrial tachycardia. However, algorithms that carefully compare atrial and ventricular rates and their relation to each others are able to discriminate between SVT and VT without delay. When SMART Detection measures an interval in the VT zone and determines that the atrial rate is faster than the ventricular rate a double tachycardia is suspected. To classify the rhythm further, the algorithm looks at ventricular stability and multiplicity. A ventricular beat is classified as atrial flutter (AFlut marker) if the atrial rate is faster than the ventricular rate, the ventricular rate is stable, and an $N: 1$ relationship exists between atrial and ventricular rates. The algorithm assumes that the accelerated ventricular rate results from atrial flutter with some degree of AV block. An AFlut interval will decrement the VT counter by one (- Fig. 21, branch 2).

\section{Atrial tachycardia or atrial fibrillation with VT}

A double tachycardia is identified along several branches of the SMART Detection 

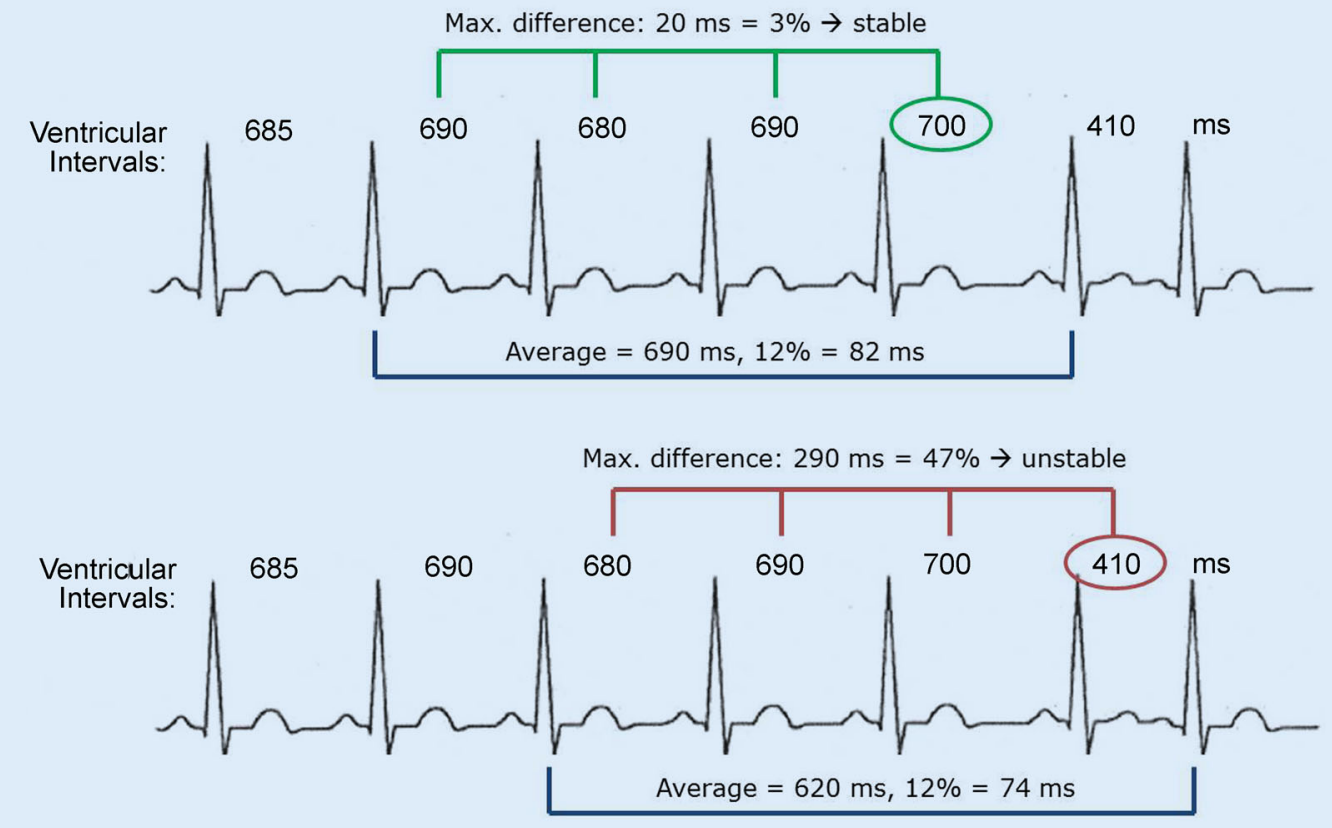

Fig. $18<$ Calculation of ventricular stability with dual-chamber detection

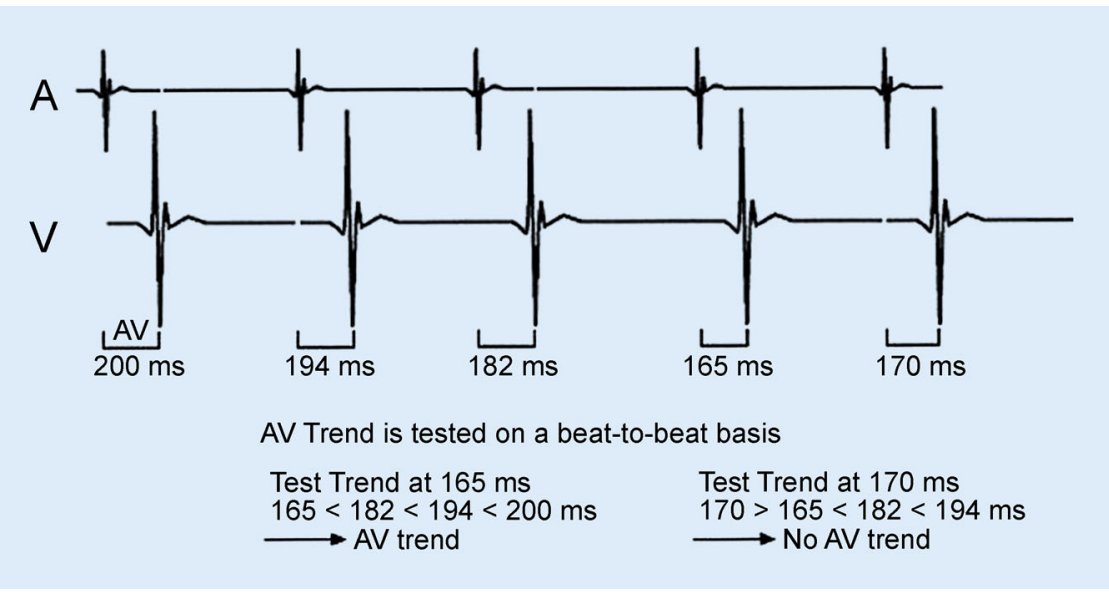

Fig. $19 \Delta$ Calculation of AV trend. The AV interval of 165 ms is tested for AV trend. There is a consistent trend in decreasing $A V$ intervals starting three intervals before the recent $A V$ interval. This $A V$ trend is not confirmed anymore with the next $\mathrm{AV}$ interval of 170 ms showing an $\mathrm{AV}$ prolongation

decision tree. The double tachycardia in this section occurs when the atrial rate is faster than the ventricular rate, the ventricular rate is stable, but $N: 1$ multiplicity cannot be established. When these conditions are met, a VT with concurrent atrial tachycardia or atrial fibrillation is identified. Each beat will be marked as VT (• Fig. 21, branch 3).

\section{Atrial fibrillation}

Atrial fibrillation is by nature irregular or chaotic with atrial intervals of varying duration. In turn, these atrial intervals are conducted irregularly to the ventricles. SMART Detection identifies irregular conduction and declares atrial fibrillation when the atrial rate is faster than the ventricular rate and the ventricular rate is unstable. Each ventricular interval is marked with an AFib marker. Therapy is withheld for this episode. An event declared as atrial fibrillation will decrease the VT counter by four to reduce the potential risk of delivering inappropriate therapy (• Fig. 21, branch 4).

\section{VT with unstable atrial arrhythmia}

Discrimination of VT is most difficult when atrial and ventricular rates are equal. Therefore, SMART Detection branches out to identify six different classification pathways when the atrial and ventricular rates are equal. When the algorithm identifies equal atrial and ventricular rates, the stability is tested in both chambers. If the ventricular rate is stable and the atrial rate is unstable, the algorithm concludes that the rhythms are dissociated. Therapy will be delivered for the VT (• Fig. 21, branch 5).

\section{Concurrent atrial tachycardia with VT}

When atrial and ventricular rates are similar and stable, several rhythms may exist. If successive AV intervals are present and strictly increase or decrease in duration (AV Trend), then one can deduce that the atrial and ventricular rhythms are close in rate, but dissociated. This small but consistent difference between the rates causes the AV interval to gradually shrink or expand over time (• Fig. 21, branch 6).

\section{Distinguishing VT from sinus tachycardia}

When atrial and ventricular rates are similar and stable with no AV trend, 


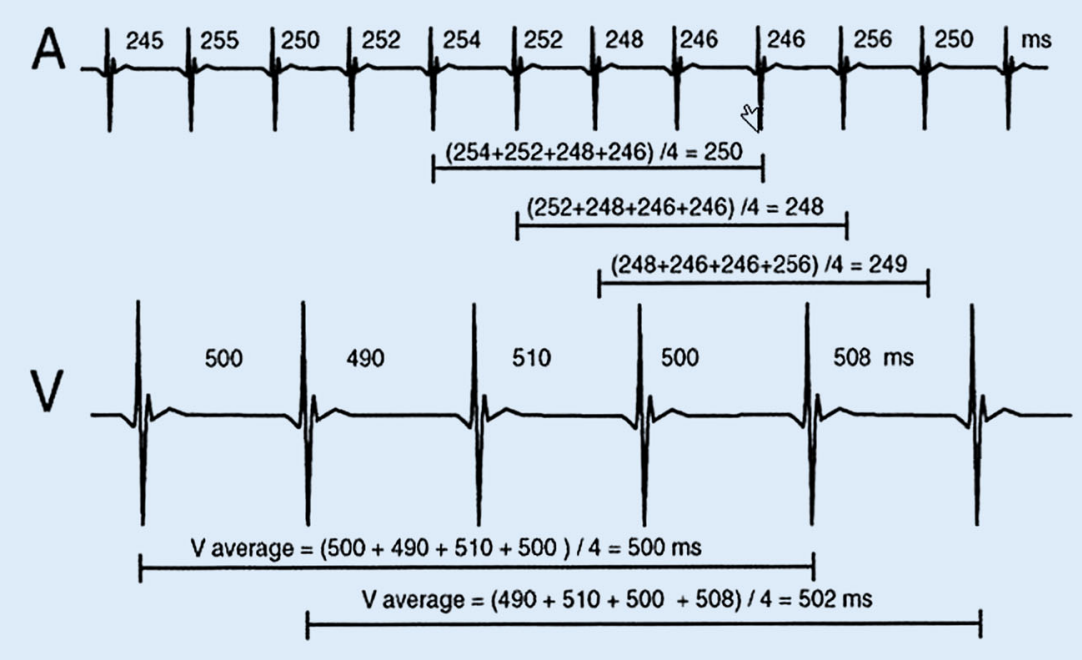

Fig. $20 \Delta$ Evaluation of Multiplicity. The ventricular average calculated on the fifth ventricular event is $500 \mathrm{~ms}$. The corresponding atrial average interval is $250 \mathrm{~ms}$. Consequently, Multiplicity is established for this event. As detection continues, each new interval is tested to determine whether the 2:1 pattern exists with a tolerance of $12 \mathrm{~ms}$

SMART Detection branches out to identify two different rhythms; monomorphic VT with 1:1 retrograde conduction and sinus tachycardia with 1:1 antegrade conduction. Monomorphic VT often starts rapidly with a significant rate increase occurring over three or four beats. In contrast, sinus tachycardia may develop over a longer time period such as during exercise. The sudden onset criterion is used to distinguish monomorphic VT with retrograde conduction from sinus tachycardia (• Fig. 21, branch 7 and 8). Intervals classified as Sinus Tachycardia are labeled with SinusT marker.

\section{Polymorphic VT with retrograde conduction}

Polymorphic VT can occur at higher rates than monomorphic VT. In addition, the QRS morphology and the heart rate can vary. When polymorphic VT is conducted back to the atrium on a beat-tobeat basis, instability is present in both chambers. Since the retrograde conduction is consistent and the intervals are unstable, the AV intervals become irregular. A polymorphic VT with retrograde conduction is detected when atrial and ventricular rates are equal, both chambers are unstable, and the AV interval is stable. Therapy is initiated to treat and terminate the polymorphic VT (• Fig. 21, branch 9).

\section{Multifocal atrial tachycardia with antegrade conduction}

If the atrial and ventricular rates are equal, both rhythms are unstable, but the AV intervals are regular, then a direct association between atrial and ventricular rhythms can be suggested. In other words, the rhythm originates in the atrium, and therefore the rhythm is irregular atrial tachycardia with 1:1 antegrade conduction. In this case, each beat is labeled with 1:1 marker, and therapy is appropriately withheld. An episode of atrial tachycardia is declared after twice the programmed VT count is met (• Fig. 21, branch 10).

\section{SMART redetection}

This is used after a VT therapy delivery to withhold further therapy if the delivered therapy resulted in an SVT. VT redetection is based on the programmable redetection count. The sustained VT timer is not utilized when SMART Redetection is $\mathrm{ON}$.

\section{Therapy reset}

The purpose of Therapy Reset (also called Forced Termination) is to reset therapies after a successful termination of a VT that results in an SVT (e. g., permanent AFib). In this way, all programed therapies will be available should the arrhythmia revert back to a VT/VF. If an SVT occurs af- ter successful termination of VT/VF and SMART Redetection is used, no therapy will be delivered for the SVT during redetection. If the SVT is still ongoing when the forced termination timer expires, the device will declare the end of the episode. The device will then record a new SVT episode if the SVT is still present and SVT EGM storage is ON.

\section{Monitoring zone}

In addition to programming therapies for VT zones, you may program a Monitoring Zone in the VT1 zone only. A monitoring zone cannot be programmed in the VT2 zone. The monitoring zone may be used tolook for nonsustained VTs. Monitoring zones are programmed the same as standard VT1 zone programming except that therapies are not provided. Monitoring zone programs may also include the programming of SMART Detection as well to look for SVTs that may be previously known.

\section{Conclusion}

ICDs by BIOTRONIK provide a sophisticated sensing algorithm that can be flexibly adjusted in case of over- or undersensing situations during clinical troubleshooting. However, VF detection should be retested when sensing settings are reprogrammed. Classification of cardiac signals is accomplished primarily by measuring cardiac cycle length. These are ventricular intervals in singlechamber detection and ventricular, atrial and $\mathrm{A}-\mathrm{V}$ intervals in dual-chamber detection. In addition, the ICD can also utilize abrupt changes in rate, irregularity of cycle lengths and other criteria as morphology to further differentiate tachyarrhythmias. To achieve best clinical results, the programming should be individualized after the occurrence of clinical episodes for each patient. Programming can be challenging in patients with atrial fibrillation and rapid ventricular response, slow VT that starts during exercise-induced sinus tachycardia, or onset of an AV nodal reentry tachycardia. Clinical studies demonstrated that the SMART Detection provided $100 \%$ sensitivity and $89 \%$ specificity [2]. 


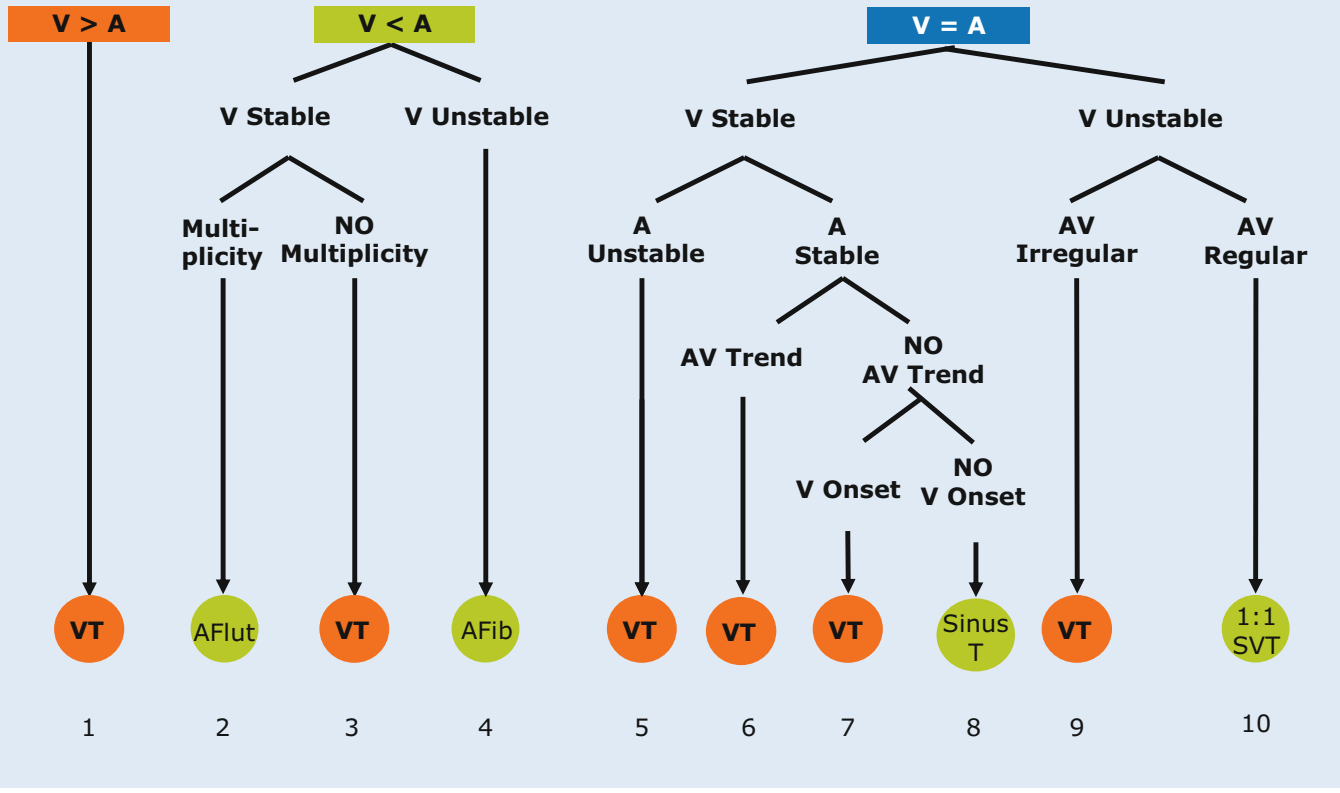

Fig. $21<$ Decision tree of the SMART Detection algorithm. If the ventricular rate falls into VT zone and the ventricular rate is faster than the atrial rate the VT counter is increased. If the ventricular rate falls into VT zone and the atrial rate is faster than the ventricular rate additional detection criteria like Ventricular Stability and Multiplicity are considered. When ventricular and atrial rates are equal, discrimination of tachyarrhythmias is more difficult. Additional detection criteria are used to identify double tachycardia, sinus tachycardia, and VT with retrograde conduction, among other rhythms

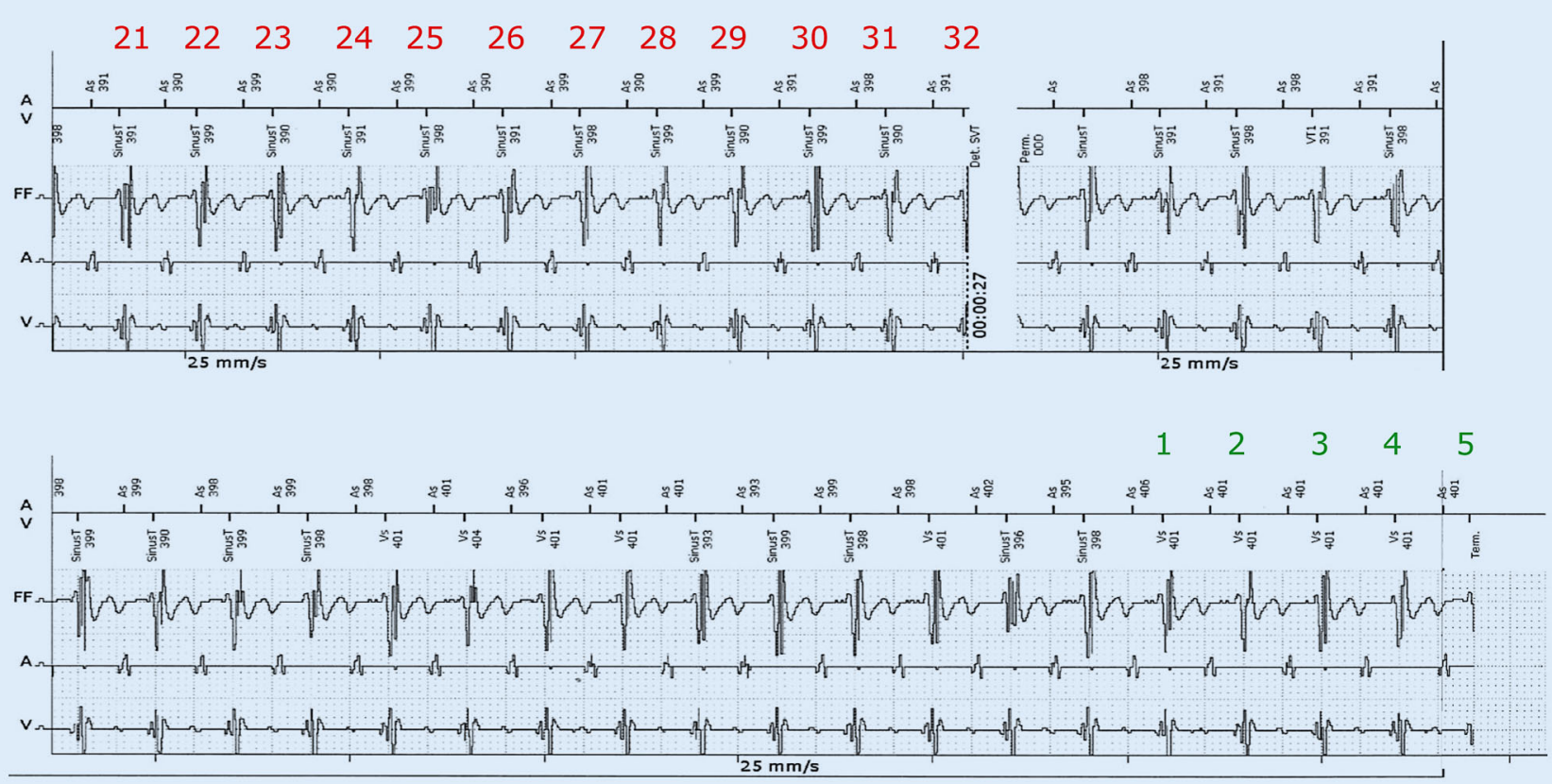

Fig. $22 \Delta$ Example of detection and termination of a sinus tachycardia in the SVT. A VT zone is programmed at $400 \mathrm{~ms} / 150$ bpm with a detection counter of 16 intervals. The dual chamber detection algorithm SMART classifies this tachyarrhythmia as sinus tachycardia (SinusT). SVT detection is fulfilled (Det. SVT, dotted line) when 32 consecutive intervals (i.e., double the VT counter, red numbers) are reached. The duration (00:00:27) indicates that the tachycardia continues for another $27 \mathrm{~s}$. The termination of the episode (Term.) is declared when 5 consecutive ventricular-sensed events (Vs, green numbers) are observed. This corresponds to so-called "short termination" 


\section{Corresponding address}

\section{Dipl.-Ing. T. Brüggemann}

Global Education and Training, Cardiac Rhythm Management, BIOTRONIK SE \& Co. KG

Woermannkehre 1, 12349 Berlin, Germany

thomas.brueggemann@biotronik.com

\section{Compliance with ethical guidelines}

Conflict of interest. T. Brüggemann, D. Dahlke, A. Chebbo and I. Neumann state that they have no competing interest.

This article does not contain any studies with human participants or animals performed by any of the authors.

\section{References}

1. http://web.hrsonline.org/Health-Policy/ClinicalDocuments/Appendix-B-Manufacturer-SpecificTranslation-of-Recommendations.pdf in relation to $2015 \mathrm{HRS} / \mathrm{EHRA} / \mathrm{APHRS} / \mathrm{SOLAECE}$ Expert Consensus Statement on Optimal Implantable Cardioverter-Defibrillator Programming and Testing. Publication Date: November 19, 2015

2. Sinha AM, Stellbrink C, Schuchert A, Möx B, Jordaens $L$, Lamaison $D$, Jaswinder $G$, Kaplan A, Merkely B (2004) Clinical experience with a new detection algorithm for differentiation of supraventricular from ventricular tachycardia in a dual-chamber defibrillator. J Cardiovasc Electrophysiol 15:646-652 